\title{
Comment on: A pilot study of ruxolitinib as a front-line therapy for 12 children with secondary hemophagocytic lymphohistiocytosis
}

\author{
Huan $\mathrm{Xu}^{1}$ and Hongbo Chen $^{1}$ \\ ${ }^{1}$ Wuhan Union Hospital
}

November 30, 2020

Title: Comment on: A pilot study of ruxolitinib as a front-line therapy for 12 children with secondary hemophagocytic lymphohistiocytosis

Authors: Huan $\mathrm{Xu}^{1}$, Hongbo Chen ${ }^{1}$

Affiliations: ${ }^{1}$ Department of Pediatrics, Union Hospital, Tongji Medical College, Huazhong University of Science and Technology, Wuhan, China

Correspondence: Hongbo Chen, Address: Department of Pediatrics, Union Hospital, Tongji Medical College, Huazhong University of Science and Technology, 1277 Jiefang Avenue, Wuhan 430022, China, Telephone: +86-13006114930, Email: hbchen@hust.edu.cn.

Word Count for Main Text: 505

Running Heads: Ruxolitinib therapy for HLH in children

Keywords: Ruxolitinib, HLH, therapy

\begin{tabular}{ll}
\hline Abbreviations & Full term or phrase \\
HLH & hemophagocytic lymphohistiocytosis \\
CR & complete response \\
JAK & Janus kinase \\
EBV & Epstein-Barr virus \\
EFS & event-free survival \\
\hline
\end{tabular}

In a recent article in Haematologica, Zhang and colleagues highlighted the efficacy and safety of ruxolitinib and the possibility of ruxolitinib-based front-line therapy in pediatric patients with secondary hemophagocytic lymphohistiocytosis $(\mathrm{HLH})^{1}$. The rapid recovery of clinical manifestations and normalization of clinical laboratory indexes demonstrated that ruxolitinib could serve as a potential front-line treatment option for secondary HLH in children, consequently reducing the toxicity compared to intense chemotherapy.

Although HLH-1994/2004 regimen has been recommended to the treatment of HLH in recent decades, there are still some patients who do not respond well or have intolerable side effects from conventional chemotherapies $^{2,3}$. To date, there are many case reports about the experiences of utilizing Janus kinase (JAK) $1 / 2$ inhibitor, ruxolitinib, in the treatment of $\mathrm{HLH}^{4,5}$. However, most of these cases involved adults, and few cases have been reported about the use of ruxolitinib in pediatric patients with HLH. The study firstly enrolled 12 children with HLH, and evaluated the safety and efficacy of ruxolitinib in pediatric patients. They found that the overall response rate of ruxolitinib was $83.3 \%$, with $66.7 \%$ in complete response (CR). More importantly, no serious adverse effects were observed in this study, except for some grade 1-2 
gastrointestinal adverse events (gastritis, nausea, and loss of appetite) in two patients. They also brought up a new idea that clinicians could attempt to utilize ruxolitinib first for approximately 3 days to determine the treatment response and sort applicable patients to avoid chemotherapy as possible. And they found that those patients who poorly responded to ruxolitinib after 3-7 days of treatment all responded well to the subsequent HLH-1994 protocol. HLH-1994/2004 regimen and ruxolitinib can be two complementary front-line therapy strategies, and further studies are warranted to testify this idea and sort the specific HLH group sensitive to ruxolitinib to guide effective treatment in the future.

Epstein-Barr virus (EBV)-associated HLH accounts for approximately $60 \%$ of all pediatric HLH patients in China, and most of those patients have a poor response to the standard treatment of HLH-1994/2004 and have a poor prognosis ${ }^{6}$. Moreover, current ongoing clinical trials studying the use of ruxolitinib in HLH patients have not yet enrolled the patients with (EBV)-associated HLH. The study enrolled 8 pediatric patients with HLH and found that the response rate of EBV-associated HLH patients was 100\% and the CR rate was $75 \%$, and EBV DNA load in plasma of all EBV-associated HLH patients decreased dramatically within one week. All of above suggested the great possibility of ruxolitinib for treating EBV-associated HLH, though the sample size was too small.

To truly improve the clinical treatment outcome for HLH in children, further trials will require a large cohort size, coherent eligibility criteria and dosing schedules to evaluate the safety and efficacy of ruxolitinib in treating HLH in children patients, especially EBV-associated HLH. Nonetheless, this reported pilot study made a major progress in managing HLH in children patients without traditional chemotherapy.

Conflict of Interest statement: The authors declared that they have no conflicts of interest to this work.

Acknowledgements: This work is supported by the National Natural Science Foundation of China (No. 31701207).

\section{References:}

1. Zhang Q, Wei A, Ma HH, Zhang L, Lian HY, Wang D, et al. A pilot study of ruxolitinib as a front-line therapy for 12 children with secondary hemophagocytic lymphohistiocytosis. Haematologica. 2020 Jul 30. doi: 10.3324/haematol.2020.253781. [Epub ahead of print]

2. Trottestam H, Horne A, Aricò M, Egeler RM, Filipovich AH, Gadner H, et al. Chemoimmunotherapy for hemophagocytic lymphohistiocytosis: long-term results of the HLH-94 treatment protocol. Blood. 2011;118(17):4577-84.

3. Henter JI, Horne A, Aricó M, Egeler RM, Filipovich AH, Imashuku S, et al. HLH-2004: Diagnostic and therapeutic guidelines for hemophagocytic lymphohistiocytosis. Pediatric blood \& cancer. 2007;48(2):124-31.

4. Ahmed A, Merrill SA, Alsawah F, Bockenstedt P, Campagnaro E, Devata S, et al. Ruxolitinib in adult patients with secondary haemophagocytic lymphohistiocytosis: an open-label, single-centre, pilot trial. The Lancet Haematology. 2019;6(12):e630-e7.

5. Wang J, Wang Y, Wu L, Wang X, Jin Z, Gao Z, et al. Ruxolitinib for refractory/relapsed hemophagocytic lymphohistiocytosis. Haematologica. 2020;105(5):e210-e2.

6. Wang J, Wang Y, Wu L, Zhang J, Lai W, Wang Z. PEG-aspargase and DEP regimen combination therapy for refractory Epstein-Barr virus-associated hemophagocytic lymphohistiocytosis. Journal of hematology \& oncology. 2016;9(1):84. 